\title{
Effect of hydroalcoholic and aqueous extracts of Dracocephalum kotschyi on bleomycin induced pulmonary fibrosis
}

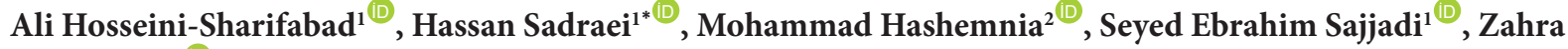 \\ Mirdamadi $^{1}$ \\ ${ }^{1}$ Isfahan Pharmaceutical Sciences Research Centre, School of Pharmacy and Pharmaceutical Sciences, Isfahan University of Medical Sciences, Isfahan, \\ I.R. Iran \\ ${ }^{2}$ Department of Pathology, Faculty of Veterinary Medicine, Razi University, Kermanshah, I.R. Iran
}

\section{A R T I C L E I N F O}

Article Type:

Original Article

\section{Article History:}

Received: 1 April 2020

Accepted: 7 July 2020

\section{Keywords:}

Dracocephalum kotschyi

Fibrosis

Inflammation

Malondialdehyde

Pirfenidone

Hydroxyproline

\begin{abstract}
A B S T R A C T
Introduction: Dracocephalum kotschyi is an Iranian traditional medicine with antiinflammatory and bronchodilatory properties. The objective of this research was to investigate the preventive effect of $D$. kotschyi extract in bleomycin induced fibrosis.

Methods: Fibrosis was induced by intratracheal administration of a single dose of bleomycin $(5 \mathrm{mg} / \mathrm{kg})$ in rat. One group received daily administration of normal saline. Other groups were treated daily with oral administration of either hydroalcoholic or aqueous extract of $D$. kotschyi $(20,40, \& 80 \mathrm{mg} / \mathrm{kg})$. Another group was treated with pirfenidone $(100 \mathrm{mg} / \mathrm{kg})$. After 4 weeks daily treatments, the animals were sacrificed and the whole lungs were dissected out for biochemical and histopathological examinations. The biochemical examination included assessment of hydroxyproline and malondialdehyde levels.

Results: The lung tissues in bleomycin treated groups showed severe tissue injuries. However, lung tissues in the groups that received hydroalcoholic or aqueous extracts of D. kotschyi showed mild to moderate tissue injuries. Intratracheal instillation of bleomycin significantly increased hydroxyproline and malondialdehyde biomarker levels compared with the sham group. In the positive control group treated with pirfenidone, there was a mark reduction in both hydroxyproline and malondialdehyde levels. Both hydroalcoholic and aqueous extracts of $D$. kotschyi significantly prevented elevation of hydroxyproline and malondialdehyde levels at the above oral doses in comparison to the vehicle treated control group.

Conclusion: Prevention of hydroxyproline and malondialdehyde elevation levels in this experiment indicates that $D$. kotschyi extract might be a suitable remedy for treatment of respiratory fibrosis induced by drugs such as bleomycin.
\end{abstract}

Implication for health policy/practice/research/medical education:

Dracocephalum kotschyi extracts displayed systemic anti-inflammatory and anti-fibrotic activities, and reduced biochemical and histopathological indices of pulmonary inflammation and fibrosis. Therefore, it is might be useful for prevention of idiopathic pulmonary fibrosis.

Please cite this paper as: Hosseini-Sharifabad A, Sadraei H, Hashemnia M, Sajjadi SE, Mirdamadi Z. Effect of hydroalcoholic and aqueous extracts of Dracocephalum kotschyi on bleomycin induced pulmonary fibrosis. J Herbmed Pharmacol. 2021;10(2):209217. doi: 10.34172/jhp.2021.23.

\section{Introduction}

Idiopathic pulmonary fibrosis is a progressive and usually fatal chronic interstitial lung disease with very limited treatment options (1). Fibrosis occurs due to the large accumulation of extracellular matrix proteins. These proteins include collagen, elastic fibers, fibronectin and laminin, among which collagen is the most abundant (2). The disease is characterized by abnormal activity of epithelial cells, in which over excretion of mediators causes overcrowding of fibroblasts followed by excessive accumulation of extracellular matrix and eventually destruction of the lung tissue (2). Acute distress syndrome 
caused by acute lung injures and traumas results in chronic interstitial pulmonary fibrosis, which is characterized by progression of fibrosis and collagen deposition (3). The pathogenic mechanism of fibrosis is not clear (4). Studies have shown that aging, smoking, environmental and occupational factors are important known risk factors $(5,6)$. Viral infections, genetic factors, exposure to paraquat, toluene, certain drugs such as methotrexate, amiodarone and bleomycin have also been identified as contributing factors $(6,7)$. With the increasing prevalence of cancers, especially solid tumors, bleomycin is used as one of the drugs in the chemotherapy regimen (8). The pulmonary fibrosis complication of this drug is high. Glucocorticoids and immunosuppressive drugs are common treatment for idiopathic pulmonary fibrosis (9). However, treatment of fibrosis with immunosuppressive drugs is not satisfactory and in most cases accompanied with serious adverse effects (10). Considering the cost of current drug treatments and their side effects, alternative and more effective therapies are required. One approach is use of herbal medicine with known active constituents with anti-fibrosis activity (11).

Dracocephalum kotschyi Boiss is one of the native plants of Iran locally known as Zarringiah (12). D. kotschyi belongs to Lamiaceae family and contains numerous flavonoids constituents with antioxidant properties (13). In traditional medicine, this herb has been used as a medicinal plant for the treatment of rheumatism and gastrointestinal problems (13). Several separate researches have reported that D. kotschyi possess immunomodulatory, anticancer, anti-hyperlipidemia, antidiabetic, anti-inflammatory and antispasmodic properties (14-20). D. kotschyi is enriched in various constituents including essential oils and flavonoids $(21,22)$. The main substances found in D. kotschyi essential oil include a-pinene, neral, geraniol, a-citral, limonene, cyclononadiene, terpinene-4-ol linalool, carveol, myrcene, germacrene-D, isopinocarveol, and a-terpineol (23). The constituents of hydroalcoholic extract, include calycopterin, xanthomicrol, isokaempferide, luteolin, apigenin, luteolin-7-O-betaD-glucopyranoside, lutcolin-3'-O-beta-D-glucuronide, apigenin-4'-O-beta-D-glucopyranoside, acacetin-7-Obeta-D-glucopyranoside and rosmarinic acid (22). Both the essential oil and hydroalcoholic extract of $D$. kotschyi have strong spasmolytic and anti-inflammatory activities $(24,25)$. Limonene and $\alpha$-terpineol are responsible for the anti-nociceptive properties of the essential oil of D. kotschyi leaf (26). Also, methoxylated flavones such as apigenin, luteolin, isokaempferid, cirsimaritin, calycopterin, penduletin and xanthomicrol have been reported to be responsible for its anticancer effects $(15,27$ 30). Luteolin has multiple biological properties such as anti-inflammatory, antioxidant and anticancer activities (31-33). In animal studies, luteolin clearly inhibited the growth and proliferation of lung fibroblasts (34) and demonstrated strong anti-fibrotic activity both in vitro and in vivo (35). Apigenin has anti-inflammatory and antioxidant properties (20). It also has cytostatic and cytoprotective activities (29,30). In a separate study, the anti-inflammatory and anti-fibrotic effects of apigenin on fibrotic lungs induced by bleomycin were demonstrated (36). As it was mentioned above, apigenin and luteolin are abundant flavonoids compounds exist in D. kotschyi (22). D. kotschyi extracts have been reported to have bronchodilatory effects on rabbit tracheal (37). In addition to anti-inflammatory and anti-fibrotic activities both apigenin and luteolin also have bronchodilatory properties (37). Thus, it is likely that D. kotschyi extract has anti-inflammatory and anti-fibrotic activities on pulmonary fibrosis. Therefore, the main objective of this research was to investigate anti-inflammatory and antifibrotic effects of hydroalcoholic and aqueous extracts of D. kotschyi in animal models.

\section{Methods and Materials}

\section{Extract preparations}

Zarringiah was collected from cultivated farm belong to Rahnama Kesht Pertikan Company located in the Shahankooh in Fereidounshahr (Isfahan-Iran). The plant was identified by the botanist Mr. Mohammad Isfa from Department of Natural Resources of Isfahan province as D. kotschyi. A voucher of the plant has been deposited in School of Pharmacy and Pharmaceutical Sciences herbarium (No: 1519). The leafy branches of the plant were dried in shade and grinned into fine powder with a grinder (Keep, Korea). Plant extraction was performed by decoction and maceration techniques $(38,39)$.

For preparation of aqueous extract, in a large baker 750 $\mathrm{mL}$ of water was added into $30 \mathrm{~g}$ of dried powder and brought to boil and then simmer for up to 1 hour until the volume was reduced to one third. The whole content was strained through Buchner funnel and then the collected liquid was concentrated by rotary apparatus (Heidolph, Germany). The concentrated extract was then freeze dried and the extract yield was calculated.

For preparation of hydroalcoholic extract the dried powder was moist with $70 \%$ ethanol and kept in a sealed container for 2 hours. The moistened plant powder was transferred to percolator and immersed in $70 \%$ ethanol (1:8 ratio) for three days accompanied by gentle shaking. After three days, the extract aliquant was removed. This process was repeated two more times by adding 70\% ethanol to the percolator. The collected extract was concentrated by rotary apparatus.

\section{Drug and solutions}

Lyophilised bleomycin sulphate (15000 IU equivalent to a standardised biological activity of $15 \mathrm{mg}$ bleomycin) (Bleo-Cell vial, Germany) was dissolved in $0.9 \%$ normal saline as $5000 \mathrm{IU} / \mathrm{mL}$ stock solution for immediate use. 
Formaldehyde was diluted with water to produce $10 \%$ solution. Hydroalcoholic extract of D. kotschyi was dissolved in $20 \%$ dimethyl sulfoxide (DMSO) in normal saline to give $8 \mathrm{mg} / \mathrm{mL}$ stock solution. Aqueous extract and pirfenidone were prepared in normal saline as $8 \mathrm{mg} /$ $\mathrm{mL}$ and $2 \mathrm{mg} / \mathrm{mL}$ stock solutions, respectively. Further dilution was made with normal saline.

\section{Experimental procedure}

Fibrosis was induced in rat by intra-tracheal instillation of bleomycin. Adult and healthy male Wistar rats (180-200 g) were used for this experimental study. The animals were purchased from School of Pharmacy and Pharmaceutical Sciences animal house. Animals were anesthetized by isoflurane in a glass container $(500 \mathrm{~mL})$ under laboratory hood. A piece of cotton wood was soaked in isoflurane solution $(30 \% \mathrm{v} / \mathrm{v}$ isoflurane in propylene glycol) and placed inside the container. When the rat was tilt on its back and did not turned on its feet consider as anesthetic cut point. The animal was then removed and placed on its back and its mouth was opened up by hanging upper canine teeth with a thread. Bleomycin $(200 \mathrm{IU} / \mathrm{kg})$ was instilled inside the trachea via a metal feeding tube. All the drugs were given orally by feeding tube every day for 4 weeks. The test groups were treated with either hydroalcoholic or aqueous extract of D. kotschyi (20 $\mathrm{mg} / \mathrm{kg}, 40 \mathrm{mg} / \mathrm{kg} \& 80 \mathrm{mg} / \mathrm{kg}$ ). The control groups were treated either with normal saline or DMSO as appropriate (negative group). The positive group was treated with standard drug pirfenidone $(100 \mathrm{mg} / \mathrm{kg})$. In the sham groups, one group received no treatment at all, while other group received intra-tracheal instillation of normal saline without other treatment. At least 6 rats were used in each group. The animals were kept in a separate room in the animal house with free access to food and water at room temperature. Prior and at the end of the study all the animals were weighed.

On $28^{\text {th }}$ day, all animals were sacrificed with $\mathrm{CO}_{2}$. Then the animal's thoracic cage was opened up and both lung tissues were carefully dissected out and weighed. The lung tissues were washed with normal saline and a lob was placed in formaldehyde for histopathological examination. The other lob was frozen at $-80^{\circ} \mathrm{C}$ for assessment of biochemical parameters.

\section{Histopathological examination}

For histopathological evaluation, lung tissue samples were fixed in $10 \%$ neutral buffered formalin, embedded in paraffin, sectioned at $5 \mu \mathrm{m}$ thickness, and stained with Masson's trichrome and hematoxylin-eosin staining for light microscopic examination. The parameters that were assessed in histopathological sections consisted of vascular congestion, hemorrhage, interstitial edema, alveolar structural disturbance, infiltration of inflammatory cells and fibrosis. Description and scoring of histopathological lesions and evaluation of fibrosis changes were carried out as previously described by Greco et al (40) with some modifications (41). Briefly, 10 fields for each lung section were systematically examined using a $\times 10$ objective and each field was scored using the following grading scheme: grade 0 for normal tissue and grades $1-4$ for the presence of pulmonary inflammation and fibrosis. The severity of lesions was graded as 1 (mild), 2 (moderate), 3 (severe) and 4 (severe inflammation accompanied by total distortion of the structure). The extent of lesions was graded as 1 (>10\% of the slide), 2 (10\%-40\%), 3 (40\%-70\%), and 4 $(<70 \%)$ of tissue affected. Fields predominantly occupied by portions of large bronchi or vessels were not counted.

\section{Biochemical assessment}

In the biochemical assessment hydroxyproline was measured as an index of tissue collagen contents and malondialdehyde was assessed as an indication of lipid peroxidation factors. Hydroxyproline was measured by a standard kit (KHPA 96, Kiazist Hamedan Company, Iran) as explained in its protocol manual. Briefly, a small piece of defreeze tissue (140 to $280 \mathrm{mg}$ ) was homogenized and digested in strong acid $(12 \mathrm{M} \mathrm{HCl})$ and after oxidative reaction with chromogen the supernatant had an orange-purple color. Pure hydroxyproline was used for construction of standard curve and light absorption was read at $550 \mathrm{~nm}$ wavelength with Elisa (Elisa reader BioTek).

Malondialdehyde content of the tissue was measure by colorimetry-fluorimetry lipid peroxidation kit (KMDA-96, Kiazist Hamedan Company, Iran). Briefly, malondialdehyde was reacted with thiobarbituric acid to form a complex which gives orange color. Light absorption was read at $550 \mathrm{~nm}$ wavelength with Elisa colorimetry apparatus.

Data assessment and statistical analysis

Tissue hydroxyproline and malondialdehyde was measured from constructed standard curves and expressed as $\mathrm{mg} / \mathrm{g}$ and $\mathrm{nM} / \mathrm{g}$ tissue, respectively. Data values were expressed as mean \pm SEM (standard error of mean). For statistical comparison Student's $t$ test was used for parametric data. For non-parametric data Kruskal-Wallis test was used. GraphPad Prism computer program software was used for statistical analysis. $P$ values less than 0.05 was considered statically significant.

\section{Results}

Bleomycin was used as a tool to induce pulmonary fibrosis. In microscopic examination lung tissues in the control rats (without bleomycin) showed normal bronchial and alveolar spaces and normal thickening of alveolar septa (Figure 1). The main histopathologic findings in bleomycin group were disturbance of alveolar structure, severe necrosis and epithelial degeneration of alveolar walls 
with extensive cellular thickening of interalveolar septa, vascular congestion, interstitial edema, severe infiltration of inflammatory cells (predominantly mononuclear cells including macrophages and lymphocytes and less neutrophils) in the interstitial and peribronchiolar area along with many collagen bundles had grown into the interstitial area (Figure 1).

The pattern of lesions in the treated groups with hydroalcoholic and aqueous extracts of D. kotschyi were clearly resolved. The extent and severity of the lesions, particularly the necrosis development, inflammation and fibrosis, were markedly less severe in compared with bleomycin treated group (Figures 2 and 3). In comparison between treated groups, the most therapeutic effects were observed with the administration of hydroalcoholic extract of D. kotschyi. Generally, high dose of the extracts $(80 \mathrm{mg} /$ $\mathrm{kg}$ ) had a more preventive effect on the damaged lungs compared to lower doses (Figures 2 and 3). To confirm the effects of $D$. kotschyi extracts on the lung injuries induced by bleomycin, a semiquantitative score of the severity and extent of inflammation in the lung sections were estimated by numerical scoring (Figure 4). According to the observations made on the Masson's trichrome-

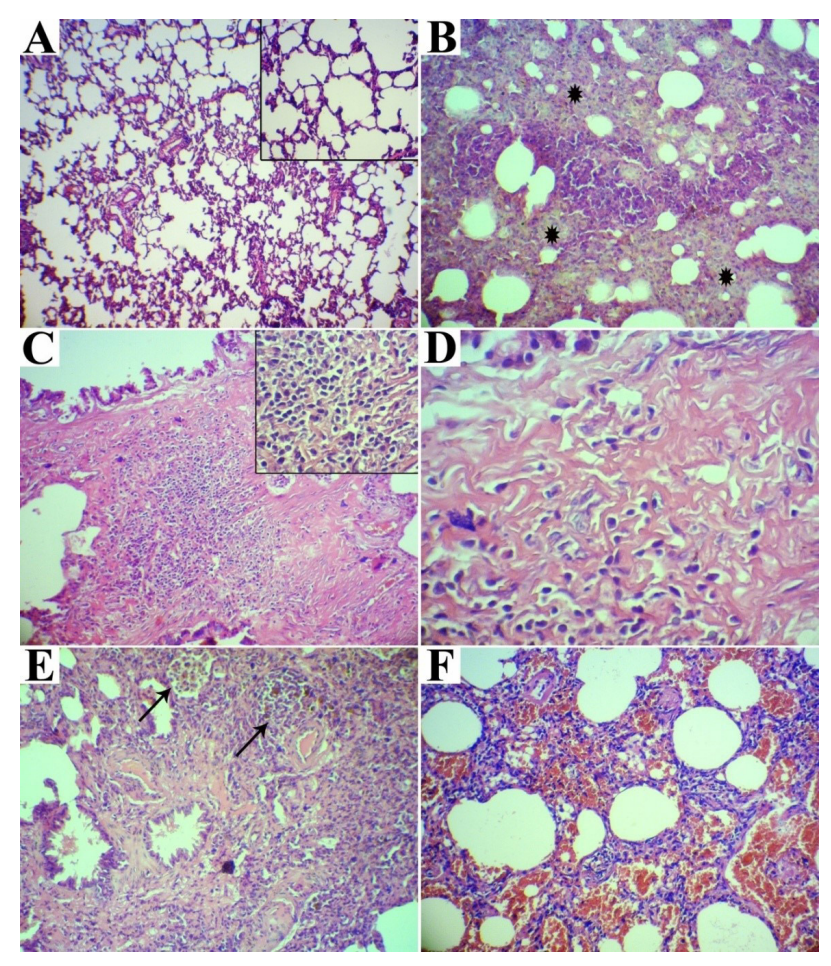

Figure 1. Microscopic features of rat lung tissue in sham $(A)$ and bleomycin treated groups (B-F). (A): Normal appearance of the lung parenchyma in sham group (HE staining; $\times 300)$. (B): Severe necrosis (stars) with extensive cellular thickening of interalveolar septa (HE staining; $\times 750)$. (C): Severe infiltration of mononuclear cells in the lung parenchyma (HE staining; $\times 750$ ). (D): Many tick collagen bundles into the interstitial area (HE staining; $\times 3000)$. (E): Increased number of alveolar macrophages (arrows) (HE staining; $\times 750)$. (F): Severe vascular congestion (HE staining; $\times 750$ ). stained sections, fibrosis score was markedly increased in the rats treated with bleomycin, but these scores were significantly reduced in the rats treated with different doses of extracts of D. kotschyi and pirfenidone (Figure 5). Similarly, the increase in inflammation score induced by bleomycin significantly ameliorated by the treatments

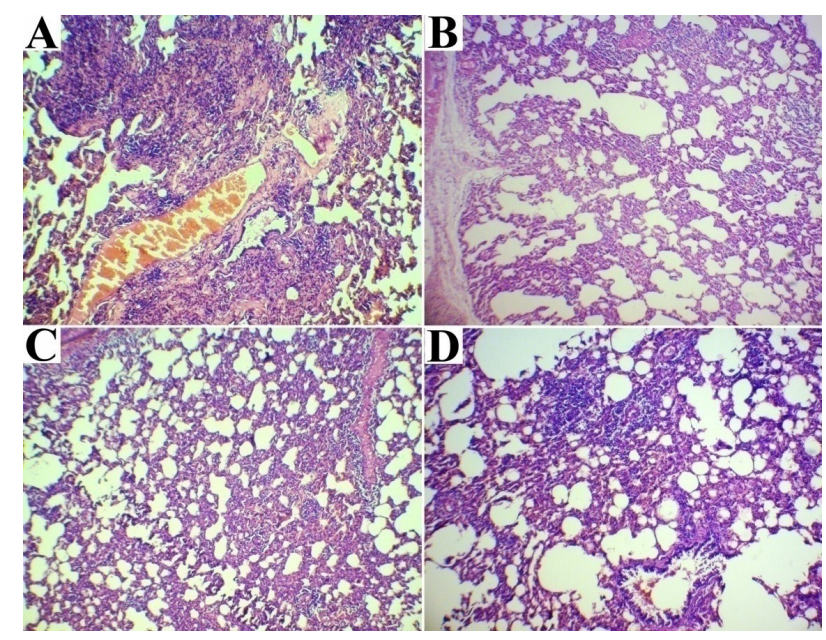

Figure 2. Microscopic features of rat lung tissue in different treated groups following intra-tracheal instillation of bleomycin (HE staining; $\times 750)$. (A): Reduction in inflammatory cell infiltration and fibrosis in the interstitial lung tissue in rat treated with hydroalcoholic extract of $D$. kotschyi $(20 \mathrm{mg} / \mathrm{kg})$. (B, C \& D): Reduction in infiltration of inflammatory cell and thickening of interalveolar septum in rat treated with aqueous extract of $D$. kotschyi $(80 \mathrm{mg} / \mathrm{kg})$, hydroalcoholic extract of $D$. kotschyi (80 $\mathrm{mg} / \mathrm{kg}$ ) and pirfenidone respectively.

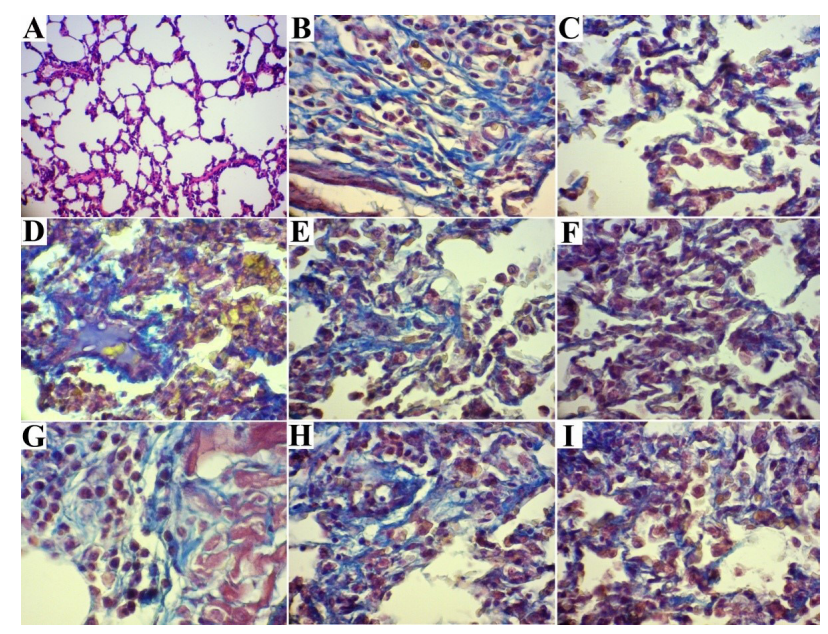

Figure 3. Masson's trichrome staining of rat lung tissues for collagen in different treated groups (HE staining; $\times 3000$ ). (A): Microscopic examination of lung tissues in the sham group (without bleomycin), showing no collagen deposition. (B): Bleomycin treated control group, showing excessive collagen deposition in the interstitial lung tissue. (C): Pirfenidone treated group, showing mild fibrosis. (D, E \& F): D. kotschyi aqueous extract treated groups $(20,40 \& 80 \mathrm{mg} / \mathrm{kg}$, respectively), showing marked reduction of interstitial deposition of collagen. (G, H \& I): D. kotschyi hydroalcoholic extract treated group (20, 40 \& $80 \mathrm{mg} /$ $\mathrm{kg}$, respectively), showing marked reduction of interstitial deposition of collagen in comparison with the bleomycin treated group. 
with pirfenidone and all doses of $D$. kotschyi extracts except with lowest doses of aqueous extract $(20 \mathrm{mg} / \mathrm{kg})$. Neither pirfenidone nor D. kotschyi extracts doses used in this study provided $100 \%$ protection against bleomycin induced inflammation and fibrosis.

Tissue hydroxyproline level was measured as an assessment of collagen infiltration in the lung tissue. Hydroxyproline is one of the constituents of collagen forming main components of the extracellular matrix. This amino acid is exclusively present in collagen, and its measurement directly indicates the amount of collagen in the tissue sample. In the sham group receiving normal saline and those without treatment, there was no significant difference between hydroxyproline contents in the lung tissues. However, in the group, which received bleomycin alone there was a sharp increase in the hydroxyproline contents (Figure 6). Hydroalcoholic extract of D. kotschyi in a concentration dependent manner reduced bleomycin induced hydroxyproline deposition (Figure 6). The hydroxyproline levels were reduced by $25 \%$, $39 \%$ and $51 \%$ with oral doses of 20,40 and $80 \mathrm{mg} / \mathrm{kg}$, respectively. Oral administration of aqueous extract also in a concentration dependent manner inhibited hydroxyproline production. The percentage reduction in hydroxyproline level was calculated as $17 \%, 33 \%$ and $46 \%$ with oral doses of 20, 40 and $80 \mathrm{mg} / \mathrm{kg}$, respectively (Figure 6). In the

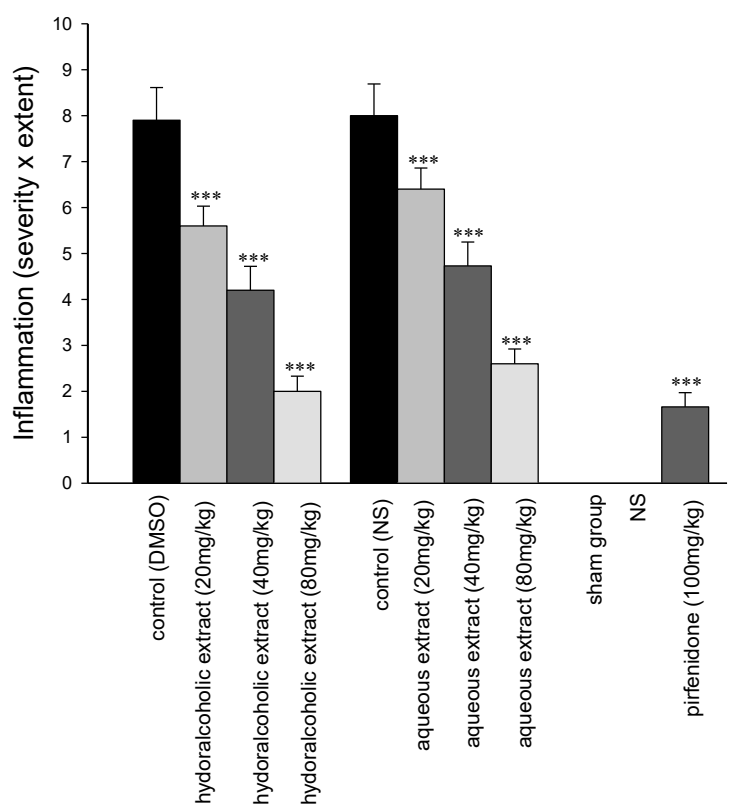

Figure 4. Numerical scoring of the severity and extent of bleomycin induced inflammation in rat lungs. On microscopic examination each field was scored using the following grading scheme: grade 0 for normal tissue and grades 1-4 for the presence of pulmonary inflammation. Inflammation was induced by intratracheal instillation of bleomycin $(5 \mathrm{mg} /$ $\mathrm{kg}$ ). D. kotschyi extracts, pirfenidone or vehicle were given orally for 4 weeks. Collected data are expressed as multiplication of severity and extent of inflammation and presented as mean \pm SEM $(n=6)$. Stars show statistically significant difference with the vehicle treated group. KruskalWallis test was used for statistical comparison $\left({ }^{\star \star *} P<0.001\right)$.

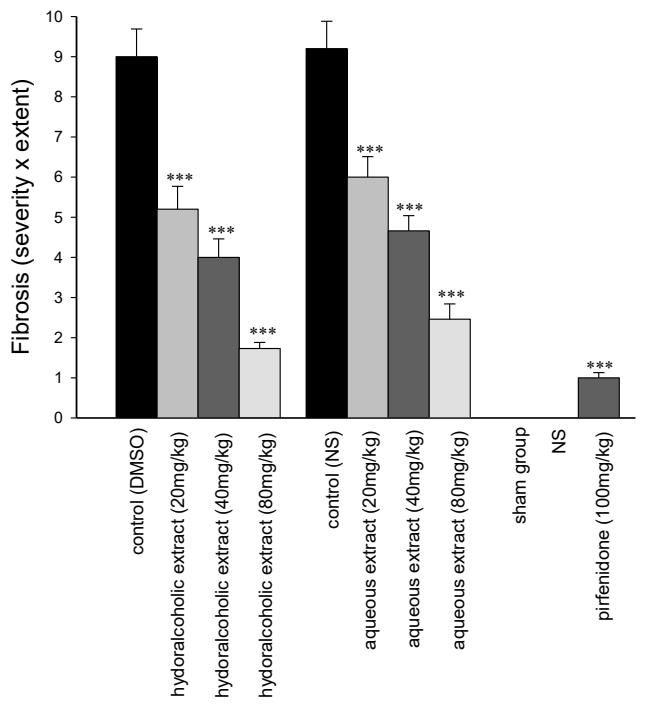

Figure 5. Numerical scoring of the severity and extent of bleomycin induced fibrosis in rat lungs. On microscopic examination each field was scored using the following grading scheme: grade 0 for normal tissue and grades 1-4 for the presence of pulmonary fibrosis. Fibrosis was induced by intratracheal instillation of bleomycin $(5 \mathrm{mg} / \mathrm{kg})$. D. kotschyi extracts, pirfenidone or vehicle were given orally for 4 weeks. Collected data are expressed as multiplication of severity and extent of inflammation and presented as mean \pm SEM $(n=6)$. Stars show statistically significant difference with the vehicle treated group. Kruskal-Wallis test was used for statistical comparison $\left({ }^{* * *} P<0.001\right)$.

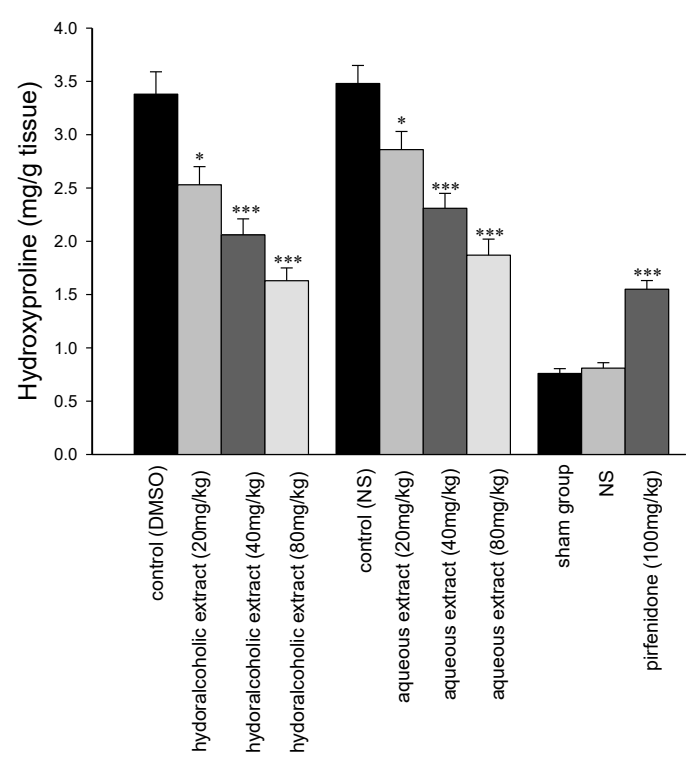

Figure 6. Hydroxyproline level assessments in bleomycin induced fibrosis in the rat lungs. Hydroxyproline level was calculated from constructed standard curves and expressed as $\mathrm{mg} / \mathrm{g}$ tissue. Fibrosis was induced by intratracheal instillation of bleomycin $(5 \mathrm{mg} / \mathrm{kg})$. D. kotschyi extracts, pirfenidone or vehicle were given orally for 4 weeks. Collected data are expressed as multiplication of severity and extent of inflammation and presented as mean \pm SEM $(n=6)$. Stars show statistically significant difference with the vehicle treated group. Student's t-test was used for statistical comparison $\left({ }^{\star} P<0.05,{ }^{* * *} P<0.001\right)$. 
positive control group, pirfenidone $(100 \mathrm{mg} / \mathrm{kg})$, the hydroxyproline level was reduced by $55 \%$ compared with the control group (Figure 6).

Amount of malondialdehyde content in the lungs represents tissue oxidative stress which is an indication for tissue inflammation. As shown in Figure 7, a residual level of malondialdehyde content in the lungs of sham group was seen in comparison to the bleomycin treated group. In the group which received normal saline instead of bleomycin there was no significant increase in malondialdehyde level (Figure 7). Daily treatment with $D$. kotschyi extracts, significantly attenuated malondialdehyde contents due to bleomycin induced inflammation (Figure 7). The inhibitory effect of both hydroalcoholic and aqueous extracts were concentration dependent. For example, the hydroalcoholic extract reduced malondialdehyde contents of the lung tissue by $41 \%, 48 \%$ and $51 \%$ with oral doses of 20,40 and $80 \mathrm{mg} / \mathrm{kg}$, respectively (Figure 7). Similar pattern of inhibition was seen with aqueous extract of $D$. kotschyi (Figure 7). Pirfenidone $(100 \mathrm{mg} / \mathrm{kg})$ inhibited malondialdehyde production by $53 \%$ in comparison with the control group (Figure 7).

\section{Discussion}

Pulmonary fibrosis develops following increase in collagen deposition and cell proliferation in the interstitial tissues (2). Fibroblasts and myofibroblasts are responsible for collagen production which results in lung functional incapability (3). Management of pulmonary fibrosis proved to be difficult with limited success. In this study bleomycin induced lung injury was used to investigate anti-inflammatory and anti-fibrotic actions of D. kotschyi extract in an animal model. Bleomycin triggers a series of immunological reactions which enhance inflammatory cells mobilization and thereby cause injury to alveolar wall (42). Furthermore, bleomycin provokes fibroblast proliferation, the activity of which, results in increased collagen deposition and fibrosis in the alveolar interstitial region (3). The pathophysiology of pulmonary fibrosis induced by intra-tracheal instillation of bleomycin involves complex interaction among pro-inflammatory factors including inflammatory cytokines interleukin$1 \mathrm{~b}$ (IL-1b), tumour necrosis factor- $\alpha$ (TNF- $\alpha$ ) and transforming growth factor- $\beta$ (TGF- $\beta$ ),). Although these factors may play crucial roles in the establishment of pulmonary fibrosis, however, multiple mediators and cell lineages are involved (42). Histological changes observed following intra-tracheal instillation of bleomycin is believed to have close resemblance to chronic obstructive pulmonary disease and idiopathic pulmonary fibrosis (43). Furthermore, pro-inflammatory cytokines have been suggested to be responsible for enhanced responses of airway smooth muscles and it is believed that they play a significant contribution into the over reactive response of airways observed in asthma (43).

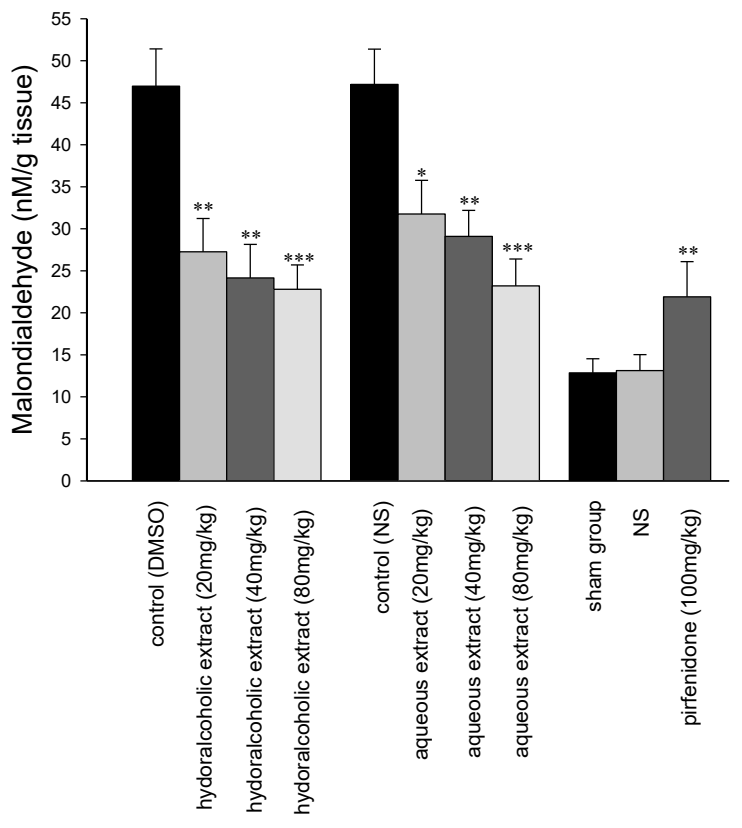

Figure 7. Malondialdehyde level assessments in bleomycin induced inflammation in rat lungs. Malondialdehyde level was calculated from constructed standard curves and expressed as $\mathrm{nM} / \mathrm{g}$ tissue. Inflammation was induced by intratracheal instillation of bleomycin $(5 \mathrm{mg} / \mathrm{kg})$. $D$. kotschyi extracts, pirfenidone or vehicle were given orally for 4 weeks. Collected data are expressed as multiplication of severity and extent of inflammation and presented as mean $\pm \operatorname{SEM}(n=6)$. Stars show statistically significant difference group with the vehicle treated group. Student's t-test was used for statistical comparison $\left({ }^{*} P<0.05,{ }^{* *} P<0.01\right.$, $\left.{ }^{\star * *} P<0.001\right)$.

Pirfenidone has been introduced as an anti-fibrotic agent (44). Pirfenidone inhibits fibroblast proliferation, reduces extracellular matrix production and prevents TGF- $\beta$ dependent collagen synthesis (45).

The pathological examinations of the lungs tissues showed that thickness of alveolar wall and development of fibrosis were reduced by co-treatment with $D$. kotschyi extracts in the bleomycin treats rats. Furthermore, $D$. kotschyi extracts reduced accumulation of hydroxyproline in the rat lungs in group which received bleomycin. Hydroxyproline is one of the collagen component mainly found in outer cell matrix (46). This amino acid is unique for collagen and its measurement directly represents sample collagen contents (47). In this research maximum increase in hydroxyproline level and malondialdehyde activity in the lung tissues were seen in bleomycin receiving group and minimal values belonged to the vehicle treated group. Increase in malondialdehyde level was associated with development of inflammatory lesions of the lungs in the bleomycin treated groups. In the group which received D. kotschyi extracts and bleomycin, there was a substantial reduction in malondialdehyde activity in comparison to vehicle treated groups. Malondialdehyde is one of the factors involved in lipid peroxidation and cause direct oxidative damage to unsaturated fatty acid in the cells (48). Malondialdehyde is considered as a reliable marker 
for assessment of inflammation in the injured tissues (48). Therefore, these results not only indicate anti-oxidant activity but clearly demonstrate the anti-inflammatory and anti-fibrotic actions of D. kotschyi extracts.

Both hydroalcoholic and aqueous extracts of D. kotschyi demonstrated relatively similar preventive effect against bleomycin elicited pulmonary fibrosis. This resemblance could be due to presence of common components. D. kotschyi extracts are enriched in flavonoids which have potential anti-inflammatory properties $(19,22)$. Flavonoids mainly exist in glycoside forms which are water soluble and found in both aqueous and hydroalcoholic extracts. In this research both extracts were administered orally and their anti-inflammatory effects on the lungs indicate good oral absorption of active components. The pharmacological activity of flavonoids is mainly seen with their aglycone forms. However, it has been reported that in the gastrointestinal tract the sugar moiety is removed and aglycone form of flavonoids is released (49). There are substantial reports that indicate some active flavonoids are inhibitors of pro-inflammatory cytokine induced chemokine expression (50). Apigenin and luteolin are two known active flavonoids components that have been identified in the D. kotschyi extracts (22). Both these flavonoids have been reported to prevent oxidative stress and inhibit expression of inflammatory factors involved in bleomycin induced fibrosis (33-36). Furthermore, apigenin and luteolin are reported as inhibitors of TGF- $\beta$, which can ameliorate features of bleomycin induced lung fibrosis $(35,51)$. Pharmacological actions of apigenin and luteolin have close resemblance to D. kotschyi extracts Hence, it is likely that flavonoids could be responsible for the anti-inflammatory and anti-fibrotic activities seen with D. kotschyi extracts. Nevertheless, contribution of other component can't be excluded and requires further investigations.

Present study shows that D. kotschyi extracts prevent collagen deposition, inhibit lipid peroxidation and malondialdehyde activity and thereby reduce pulmonary fibrosis. Anti-inflammatory actions of D. kotschyi extracts on lungs tissue, combined with their bronchodilatory effects on airways smooth muscles (37), make them suitable remedies for treatment of bronchitis asthma.

\section{Conclusion}

In animal models, $D$. kotschyi extracts displayed systemic anti-inflammatory and anti-fibrotic activities and reduced biochemical and histopathological indices of pulmonary fibrosis. Therefore, it is suggested to be used alongside of chemotherapy treatment with anti-cancer agents such as bleomycin in order to prevent development of pulmonary fibrosis. In addition, oral drug preparation from $D$. kotschyi extracts would have beneficial anti-inflammatory effect in patients with obstructive pulmonary disease and asthma.

\section{Acknowledgement}

This project was part of Pharm D. thesis of Zahra Mirdamadi as Pharm D. student at Isfahan University of Medical Sciences.

\section{Authors' contributions}

$\mathrm{AH}$ was project manager. $\mathrm{AH}$ and $\mathrm{HS}$ supervised the pharmacological studies. SES supervised extract preparation. ZM was responsible for the experimental work and analysis of data. MH was responsible for histopathological examination of lung tissues. HS was responsible for writing the paper. All authors approved the final manuscript for publication.

\section{Conflict of interests}

The authors declare no conflict of interests.

\section{Ethical considerations}

Animal care and experiments were performed in accordance with the guidelines for the care and use of laboratory animals of the Isfahan University of Medical Sciences. The project was confirmed by the ethical committee of the university (IR.MUI.RESEARCH. REC.1398.317).

\section{Funding/Support}

This research project was financially supported by Research Department of Isfahan University of Medical Sciences (Project NO: 298037).

\section{References}

1. King TE Jr, Pardo A, Selman M. Idiopathic pulmonary fibrosis. Lancet. 2011;378(9807):1949-61. doi: 10.1016/ s0140-6736(11)60052-4.

2. Uchida M, Shiraishi H, Ohta S, Arima K, Taniguchi K, Suzuki S, et al. Periostin, a matricellular protein, plays a role in the induction of chemokines in pulmonary fibrosis. Am J Respir Cell Mol Biol. 2012;46(5):677-86. doi: 10.1165/ rcmb.2011-0115OC.

3. Kravis TC, Ahmed A, Brown TE, Fulmer JD, Crystal RG. Pathogenic mechanisms in pulmonary fibrosis: collagen-induced migration inhibition factor production and cytotoxicity mediated by lymphocytes. J Clin Invest. 1976;58(5):1223-32. doi: 10.1172/jci108576.

4. Sgalla G, Iovene B, Calvello M, Ori M, Varone F, Richeldi L. Idiopathic pulmonary fibrosis: pathogenesis and management. Respir Res. 2018;19(1):32. doi: 10.1186/ s12931-018-0730-2.

5. Baumgartner KB, Samet JM, Stidley CA, Colby TV, Waldron JA. Cigarette smoking: a risk factor for idiopathic pulmonary fibrosis. Am J Respir Crit Care Med. 1997;155(1):242-8. doi: 10.1164/ajrccm.155.1.9001319.

6. Macneal K, Schwartz DA. The genetic and environmental causes of pulmonary fibrosis. Proc Am Thorac Soc. 2012;9(3):120-5. doi: 10.1513/pats.201112-055AW.

7. Moore BB, Moore TA. Viruses in idiopathic pulmonary fibrosis. etiology and exacerbation. Ann Am Thorac Soc. 2015;12(Suppl2):S186-92. doi:10.1513/AnnalsATS.201502- 
088AW.

8. Watson RA, De La Peña H, Tsakok MT, Joseph J, Stoneham $\mathrm{S}$, Shamash J, et al. Development of a best-practice clinical guideline for the use of bleomycin in the treatment of germ cell tumours in the UK. Br J Cancer. 2018;119(9):1044-51. doi: 10.1038/s41416-018-0300-x.

9. Bendstrup E, Hilberg O, Hyldgaard C. Idiopathic pulmonary fibrosis-diagnosis and treatment. Gen Med (Los Angel). 2015;3(1):161. doi: 10.4172/2327-5146.1000161.

10. Stucker F, Ackermann D. [Immunosuppressive drugs - how they work, their side effects and interactions]. Ther Umsch. 2011;68(12):679-86. doi: 10.1024/0040-5930/a000230.

11. Yang J, Cui Y, Kolb M. How useful is traditional herbal medicine for pulmonary fibrosis? Respirology. 2009;14(8):1082-91.doi: 10.1111/j.1440-1843.2009.01644.x.

12. Rechinger KH. Dracocephalum. In: Iranica F, Rechinger KH, ed. Akademische Druck-U. Vol. 150. Verlagsanstalt Graz, Austria; 1986. 218-231.

13. Heydari P, Yavari M, Adibi P, Asghari G, Ghanadian SM, Dida GO, et al. Medicinal properties and active constituents of Dracocephalum kotschyi and its significance in Iran: a systematic review. Evid Based Complement Alternat Med. 2019;2019:9465309. doi: 10.1155/2019/9465309.

14. Amirghofran Z, Azadbakht M, Karimi MH. Evaluation of the immunomodulatory effects of five herbal plants. $J$ Ethnopharmacol. 2000;72(1-2):167-72. doi: 10.1016/s03788741(00)00234-8.

15. Jahaniani F, Ebrahimi SA, Rahbar-Roshandel N, Mahmoudian M. Xanthomicrol is the main cytotoxic component of Dracocephalum kotschyii and a potential anti-cancer agent. Phytochemistry. 2005;66(13):1581-92. doi: $\quad$ 10.1016/j.phytochem.2005.04.035.

16. Ebrahim Sajjadi S, Movahedian Atar AM, Yektaian A. Antihyperlipidemic effect of hydroalcoholic extract, and polyphenolic fraction from Dracocephalum kotschyi Boiss. Pharm Acta Helv. 1998;73(3):167-70. doi: 10.1016/s00316865(98)00016-8.

17. Pouraboli I, Nazari S, Sabet N, Sharififar F, Jafari M. Antidiabetic, antioxidant, and antilipid peroxidative activities of Dracocephalum polychaetum shoot extract in streptozotocin-induced diabetic rats: in vivo and in vitro studies. Pharm Biol. 2016;54(2):272-8. doi: 10.3109/13880209.2015.1033561.

18. Sadraei H, Asghari G, Kasiri F. Antispasmodic effect of Dracocephalum kotschyi hydroalcoholic extract on rat ileum contraction. Res Pharm Sci. 2015;10(5):446-52.

19. Kalantar K, Gholijani N, Mousaei N, Yazdani M, Amirghofran Z. Investigation of Dracocephalum kotschyi plant extract on the effective inflammatory transcription factors and mediators in activated macrophages. Antiinflamm Antiallergy Agents Med Chem. 2018;17(1):3949. doi: 10.2174/1871523017666180608081656.

20. Sadraei H, Asghari G, Khanabadi M, Minaiyan M. Antiinflammatory effect of apigenin and hydroalcoholic extract of Dracocephalum kotschyi on acetic acid-induced colitis in rats. Res Pharm Sci. 2017;12(4):322-9. doi: 10.4103/17355362.212050 .

21. Saeidnia S, Gohari AR, Hadjiakhoondi A, Shafiee A. Bioactive compounds of the volatile oil of Dracocephalum kotschyi. Z Naturforsch C J Biosci. 2007;62(11-12):793-6. doi: 10.1515/znc-2007-11-1203.
22. Gohari AR, Saeidnia S, Matsuo K, Uchiyama N, Yagura T, Ito M, et al. Flavonoid constituents of Dracocephalum kotschyi growing in Iran and their trypanocidal activity. Nat Med. 2003;57(6):250-2.

23. Yaghmai MS, Taffazoli R. The essential oil of Dracocephalum kotschyi Boiss. Flavour Fragr J. 1988;3(1):33-6. doi: 10.1002/ ffj.2730030107.

24. Sadraei H, Asghari G, Kasiri F. Comparison of antispasmodic effects of Dracocephalum kotschyi essential oil, limonene and a-terpineol. Res Pharm Sci. 2015;10(2):109-16.

25. Sadraei H, Asghari G, Alinejad M. Comparison of antispasmodic effect of hydroalcoholic extract of Dracocephalum kotschyi Boiss. in rat uterus and ileum. Res Pharm Sci. 2016;11(4):284-92. doi: 10.4103/17355362.189295.

26. Golshani S, Karamkhani F, Monsef-Esfehani HR, Abdollahi M. Antinociceptive effects of the essential oil of Dracocephalum kotschyi in the mouse writhing test. J Pharm Pharm Sci. 2004;7(1):76-9.

27. Talari M, Seydi E, Salimi A, Mohsenifar Z, Kamalinejad M, Pourahmad J. Dracocephalum: novel anticancer plant acting on liver cancer cell mitochondria. Biomed Res Int. 2014;2014:892170. doi: 10.1155/2014/892170.

28. Faham N, Javidnia K, Bahmani M, Amirghofran Z. Calycopterin, an immunoinhibitory compound from the extract of Dracocephalum kotschyi. Phytother Res. 2008;22(9):1154-8. doi: 10.1002/ptr.2382.

29. Sung B, Chung HY, Kim ND. Role of apigenin in cancer prevention via the induction of apoptosis and autophagy. J Cancer Prev. 2016;21(4):216-26. doi: 10.15430/ jcp.2016.21.4.216.

30. Czyz J, Madeja Z, Irmer U, Korohoda W, Hülser DF. Flavonoid apigenin inhibits motility and invasiveness of carcinoma cells in vitro. Int J Cancer. 2005;114(1):12-8. doi: 10.1002/ijc.20620.

31. Das M, Ram A, Ghosh B. Luteolin alleviates bronchoconstriction and airway hyperreactivity in ovalbumin sensitized mice. Inflamm Res. 2003;52(3):1016. doi: 10.1007/s000110300021.

32. Shen ML, Wang CH, Lin CH, Zhou N, Kao ST, Wu DC. Luteolin attenuates airway mucus overproduction via inhibition of the GABAergic system. Sci Rep. 2016;6:32756. doi: 10.1038/srep32756.

33. Seelinger G, Merfort I, Wölfle U, Schempp CM. Anticarcinogenic effects of the flavonoid luteolin. Molecules. 2008;13(10):2628-51. doi: 10.3390/molecules13102628.

34. Zhang GM, Gong GQ, Li LL, Yang LN, Wang Y. Effects of luteolin on the proliferation of rat lung fibroblasts. Chin J Nat Med. 2009;7(4):297-300. doi: 10.1016/s18755364(10)60022-2.

35. Chen CY, Peng WH, Wu LC, Wu CC, Hsu SL. Luteolin ameliorates experimental lung fibrosis both in vivo and in vitro: implications for therapy of lung fibrosis. J Agric Food Chem. 2010;58(22):11653-61. doi: 10.1021/jf1031668.

36. Chen L, Zhao W. Apigenin protects against bleomycininduced lung fibrosis in rats. Exp Ther Med. 2016;11(1):2304. doi: 10.3892/etm.2015.2885.

37. Sadraei H, Ghanadian SM, Asghari G, Gavahian A. Bronchodilator effect of apigenin and luteolin, two components of Dracocephalum kotschyi on isolated rabbit trachea. J Herbmed Pharmacol. 2019;8(4):281-6. doi: 
10.15171/jhp.2019.41.

38. Samuelsson G. Drugs of Natural Origin. Stockholm: Swedish Parmaceutical Press; 1999. p. 48-9.

39. McClure JW. Physiology and functions of flavonoids. In: Harborne JB, Mabry TJ, Mabry H, eds. The Flavonoids. Boston, MA: Springer; 1975. p. 970-1055. doi: 10.1007/9781-4899-2909-9_18.

40. Greco MJ, Kemnitzer JE, Fox JD, Choe JK, Kohn J, Riley DJ, et al. Polymer of proline analogue with sustained antifibrotic activity in lung fibrosis. Am J Respir Crit Care Med. 1997;155(4):1391-7. doi: 10.1164/ajrccm.155.4.9105084.

41. Cortijo J, Cerdá-Nicolás M, Serrano A, Bioque G, Estrela JM, Santangelo F, et al. Attenuation by oral $\mathrm{N}$-acetylcysteine of bleomycin-induced lung injury in rats. Eur Respir J. 2001;17(6):1228-35. doi: 10.1183/09031936.01.00049701.

42. Phan SH, Kunkel SL. Lung cytokine production in bleomycin-induced pulmonary fibrosis. Exp Lung Res. 1992;18(1):29-43. doi: 10.3109/01902149209020649.

43. Chung F. Anti-inflammatory cytokines in asthma and allergy: interleukin-10, interleukin-12, interferongamma. Mediators Inflamm. 2001;10(2):51-9. doi: 10.1080/09629350120054518.

44. Azuma A. Pirfenidone: antifibrotic agent for idiopathic pulmonary fibrosis. Expert Rev Respir Med. 2010;4(3):30110. doi: $10.1586 /$ ers.10.32.

45. Hilberg $\mathrm{O}$, Simonsen $\mathrm{U}$, du Bois R, Bendstrup E. Pirfenidone: significant treatment effects in idiopathic pulmonary fibrosis. Clin Respir J. 2012;6(3):131-43. doi: 10.1111/j.1752-699X.2012.00302.x.
46. Cleland R, Karlsnes AM. A possible role of hydroxyprolinecontaining proteins in the cessation of cell elongation. Plant Physiol. 1967;42(5):669-71. doi: 10.1104/pp.42.5.669.

47. McAnulty RJ. Methods for measuring hydroxyproline and estimating in vivo rates of collagen synthesis and degradation. Methods Mol Med. 2005;117:189-207. doi: 10.1385/1-59259-940-0:189.

48. Requena JR, Fu MX, Ahmed MU, Jenkins AJ, Lyons TJ, Thorpe SR. Lipoxidation products as biomarkers of oxidative damage to proteins during lipid peroxidation reactions. Nephrol Dial Transplant. 1996;11 Suppl 5:48-53. doi: $10.1093 / \mathrm{ndt} / 11$. supp5.48.

49. Sadraei H, Ghanadian SM, Moazeni S. Inhibitory effect of hydroalcoholic and flavonoids extracts of Dracocephalum kotschyi, and its components luteolin, apigenin and apigenin-4'-galactoside on intestinal transit in mice. J Herbmed Pharmacol. 2019;8(1):8-13. doi: 10.15171/ jhp.2019.02.

50. Ribeiro D, Freitas M, Tomé SM, Silva AM, Laufer S, Lima JL, et al. Flavonoids inhibit COX-1 and COX-2 enzymes and cytokine/chemokine production in human whole blood. Inflammation. 2015;38(2):858-70. doi: 10.1007/ s10753-014-9995-x.

51. Wójcik KA, Skoda M, Koczurkiewicz P, Sanak M, Czyż J, Michalik M. Apigenin inhibits TGF- $\beta 1$ induced fibroblastto-myofibroblast transition in human lung fibroblast populations. Pharmacol Rep. 2013;65(1):164-72. doi: 10.1016/s1734-1140(13)70974-5. 